\title{
PHENOMENOLOGICAL DESCRIPTION OF THE COULOMB ENERGIES FOR MEDIUM-HEAVY AND SUPERHEAVY NUCLEI
}

\author{
Lutostansky Yu.S., Tikhonov V.N. \\ National Research Center "Kurchatov Institute”, Moscow, Russia \\ E-mail: lutostansky@imp.kiae.ru
}

Coulomb energy $E_{\mathrm{C}}(A, Z)$ - is one of the main characteristics of the nucleus, determining its binding energy. In the experiment, may determine the difference of the Coulomb energies of neighboring nuclei isobars:

$$
\Delta E_{\mathrm{C}}(A, Z)=E_{\mathrm{C}}(A, Z+1)-E_{\mathrm{C}}(A, Z),
$$

which is obtained from the simple relations [1] of the analog resonances energies measurements in charge-exchange reactions. For the Coulomb displacement energies $\Delta E_{\mathrm{C}}(A, Z)$ systematic several approaches were used. First, is a relation:

$$
\Delta E_{\mathrm{C}}(A, Z)=a_{\mathrm{T}}(Z+0.5) A^{-1 / 3} f(A)+b_{\mathrm{T}}(\mathrm{MeV}),
$$

where $f(A)$ is radius correction function. For $f(A)=1$ the relation (2) goes into the semi-empirical Jänecke formula [2] parameterized by Anderson, C. Wong and McClure [3]. These parameters were determined from experimental data many times in different approaches [4]. In this paper we use the new database for more than 400 nuclei and focuses on medium-heavy nuclei. Approximation accuracy is not worse than $100 \mathrm{keV}$ that is better than calculations within microscopic theory [5].

In this paper also analyzes the group theory approach to the description of the Coulomb energies of medium-heavy nuclei in the framework of $S U(4)$ symmetry. In [6] were obtained for the parameters of the theory for nuclei up to $A=60$. We analyzed heavier nuclei up to $A=244$. For heavy nuclei region deformation is taken into account, which strongly affects the $\Delta E_{\mathrm{C}}$ value.

Approximation of Coulomb displacement energy $\Delta E_{\mathrm{C}}$ was interpolated for superheavy nuclei (SHN), where is no experimental data. Deformation was taken into account with the predicted parameters $\beta_{2}$ and were considered only SHN, located on the line of beta-stability. Note that for heavy nuclei $S U(4)$-symmetry should be restored [7] and analysis of $\Delta E_{\mathrm{C}}$ values was conducted in two approaches.

The work was supported by the Russian Foundation for Basic Research Grants no. 12-02-00955 and 13-02-12106 ofi-m.

1. J.Jänecke, F.D.Becchetti, A.M.VanDenBerg, et al. // Nucl. Phys. A. 1991. V.526. P.1.

2. J.Jänecke // Z. Physik. 1960. V.160. P.171.

3. J.D.Anderson, C.Wong, J.W.McClure // Phys. Rev. B. 1965. V.138. P. 615.

4. L.Zamick et al. // Phys. Rev. C. 2013. V.87. 067302.

5. Yu.V.Gaponov, Yu.S.Lutostansky // Sov. J. Nucl. Phys. 1972. V.16. P.270.

6. D.M.Vladimirov, N.B.Shul'gina // J. Nucl. Phys. 1987. V.45. P.1586.

7. Yu.V.Gaponov, Yu.S.Lyutostanskii // JETP Lett. 1973. V.18. P.75. 